\section{Colonoscopy in a patient with a giant Morgagni hernia}

Morgagni hernia is an uncommon type of diaphragmatic hernia, which usually appears in adulthood. The diagnosis is mainly made by chest radiograph and multidetector computed tomography (CT) [1]. As in this case, colonoscopy with fluoroscopic control is very helpful too. Repair can be performed either by using a transthoracic or transabdominal approach, and good results with laparoscopic transabdominal repair have been reported [2].

The case is presented of a 63-year-old man with a giant Morgagni hernia, diagnosed by CT, who underwent a preoperative colonoscopy to rule out other associated pathology and for decompression. The colonoscopy helped to assess the very large volume of the hernia.

The patient had experienced abdominal pain, cough, expectoration, and dyspnea. No abdominal trauma was mentioned. Plain abdominal radiographs showed dilated colon and bowel loops, with fluid levels, within the chest. There was diffuse abdominal distension on physical examination.

An emergency colonoscopy was done for decompression prior to surgery. The intestinal contents and gas were drained successfully. No luminal constriction sites were seen. The colonoscope progressed directly, diagonally to the right chest, reaching the level of the second rib ( $\bullet$ Fig. 1 a) and then moved down in a parallel direction ( $\bullet$ Fig. 1 b).

The patient underwent urgent laparoscopy due to aggravation of dyspnea. Laparoscopy revealed a giant Morgagni hernia which contained much of the colon; the small bowel was very dilated. The omentum was reduced into the abdomen, the hernia sac was excised, and the defect was repaired with a $12 \times 12-\mathrm{cm}$ prosthetic mesh patch. The patient was discharged without complication 3 days after the operation.

Some cases of diaphragmatic hernia are diagnosed unexpectedly during diagnostic colonoscopy [3]. Endoscopists should be aware of the possible occurrence of diaphragmatic hernias and their risk as life-threatening complications. To avoid such dangerous complications, access to radiography facilities with fluoroscopy is recommended in endoscopic units where colonoscopies are performed.

\section{Endoscopy_UCTN_Code_CCL_1AD_2AJ}

\section{Competing interests: None}
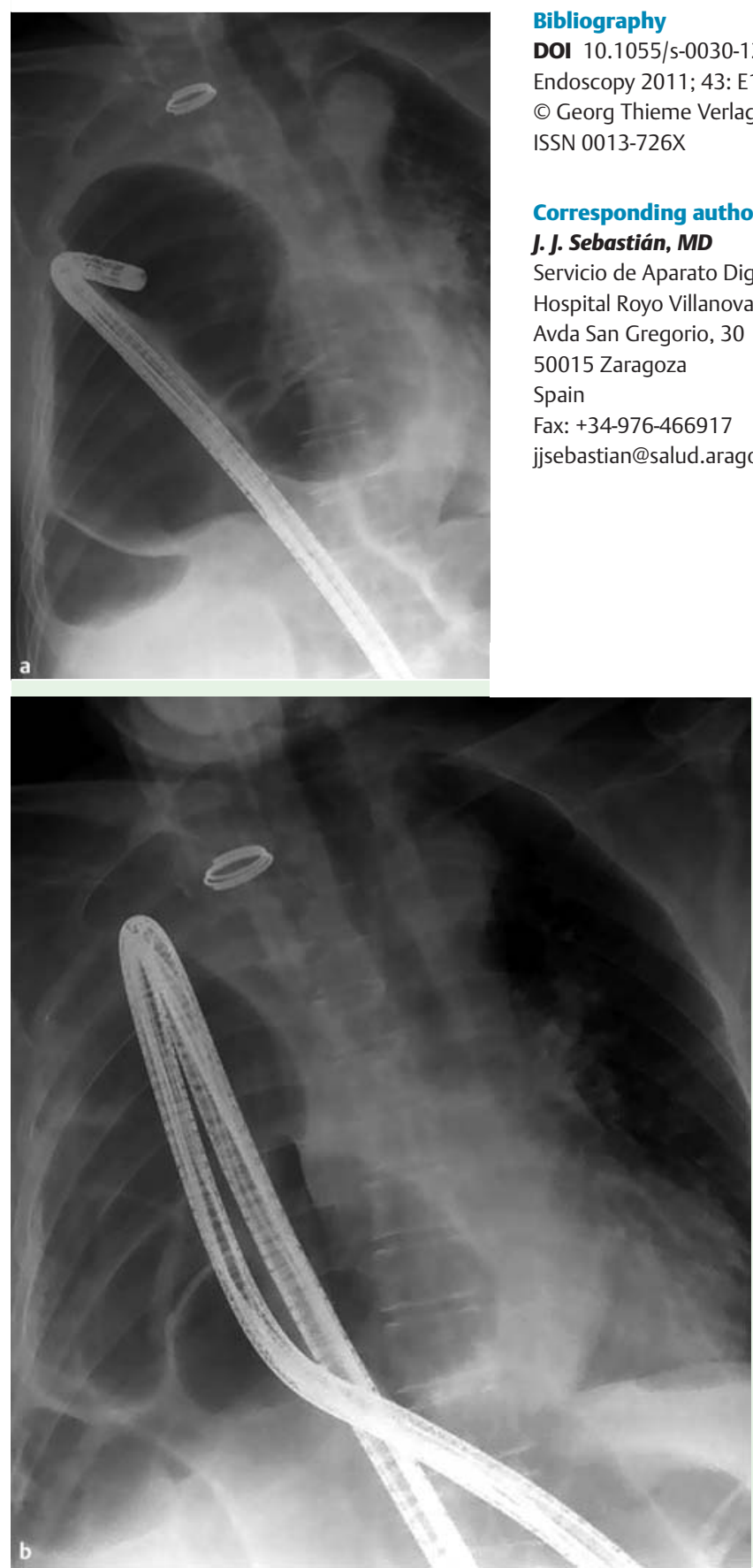

Fig. 1 a The colonoscope progressed diagonally to the right chest, reaching the level of the second rib. b The colonoscope then moved down in a parallel direction, occupying the right thorax.

\section{Corresponding author}

J. J. Sebastián, MD

Hospital Royo Villanova

Avda San Gregorio, 30

Spain

Fax: +34-976-466917

jjsebastian@salud.aragon.es 